\title{
LA TRADUCTION D'UN ALBUM ILLUSTRE DANS UN CONTEXTE UNIVERSITAIRE : ANALYSE DES TRADUCTIONS FRANÇAIS-ESPAGNOL DE YAKOUBA DE THIERRY DEDIEU
}

\author{
A TRADUÇÃO DE UM ÁLBUM ILUSTRADO NUM CONTEXTO UNIVERSITÁRIO: \\ ANÁLISE DAS TRADUÇÕES FRANCÊS-ESPANHOL DE YAKOUBA DE THIERRY \\ DEDIEU
}

\author{
THE TRANSLATION OF A PICTUREBOOK IN A UNIVERSITY CONTEXT: \\ ANALYSIS OF THE FRENCH-SPANISH TRANSLATIONS OF YAKOUBA BY \\ THIERRY DEDIEU
}

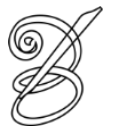 \\ Raymonda NODIS* \\ Université d'Alcalá, Espagne
}

\begin{abstract}
Résumé : La traduction d'un album illustré peut constituer une tâche complexe en raison des caractéristiques de ce type d'œuvre littéraire. Des questions telles que la relation entre le texte et l'illustration, la double page, l'oralité, le public visé, entre autres, sont quelques-uns des défis posés par la traduction d'un album. Pour cette raison, le traducteur chargé de ce type de traduction doit posséder les connaissances et les compétences requises pour produire une version adaptée à la langue et à la culture cible. Un des moyens de préparer et de former ce professionnel est par le biais d'un projet de traduction reproduisant une situation réelle de traduction littéraire, notamment à partir de la traduction d'albums illustrés. Dans cet article, nous allons étudier les problèmes rencontrés par un groupe d'étudiants de traduction, ainsi que les différentes approches qu'ils adoptent lors de la traduction du français vers l'espagnol d'un album illustré intitulé Yakouba de l'auteur-illustrateur Thierry Dedieu. Tout d'abord, nous présenterons le cadre théorique en relation avec le thème central de notre étude. Par la suite, et étant donné qu'il s'agit d'un exercice de traduction dans un contexte universitaire, nous préciserons les conditions dans lesquelles cette activité s'est déroulée et les objectifs poursuivis avec la réalisation de ce projet. Puis, nous décrirons les principales caractéristiques de l'ouvrage à traduire et enfin, nous observerons les différents niveaux auxquels les difficultés de traduction peuvent apparaître et les solutions proposées par les étudiants dans chaque cas. Tout cela nous permettra d'identifier le comportement le plus fréquent de ces traducteurs en formation et les techniques qu'ils utilisent habituellement lors de la traduction d'un album illustré.
\end{abstract}

Mots-clés : Album illustré. Traduction. Techniques de traduction. Traducteurs en formation. Contexte universitaire.

Resumo: A tradução de um álbum ilustrado pode ser uma tarefa complexa devido às características deste tipo de obra literária. Questões como a relação entre texto e ilustração, página dupla, oralidade, público-alvo, entre outros, são alguns dos desafios envolvidos na tradução de um álbum. Por este motivo, o tradutor responsável por este tipo de tradução deve possuir as competências e os conhecimentos necessários para produzir uma versão adaptada à língua e à cultura-alvo. Uma forma de preparar e formar este profissional é através de um projeto de tradução que reproduza uma situação real de tradução literária, particularmente a partir da tradução de álbuns ilustrados. No presente artigo, estudaremos os problemas enfrentados por um grupo de estudantes de tradução, bem como as diferentes abordagens que eles adotam ao traduzir um álbum ilustrado do francês para o espanhol de um álbum ilustrado intitulado Yakouba do autor-ilustrador Thierry Dedieu. Posteriormente, tratando-se de um exercício de tradução em contexto universitário, especificaremos as condições em que esta atividade foi desenvolvida e os objetivos propostos com a realização deste projeto. Em seguida, descreveremos as principais 
características da obra a ser traduzida e finalmente, observaremos os diferentes níveis em que as dificuldades de tradução podem surgir e as soluções propostas pelos alunos em cada caso. Tudo isto permitir-nos-á identificar o comportamento mais frequente destes tradutores na formação e as técnicas que habitualmente utilizam quando traduzem um álbum ilustrado.

Palavras-chave: Álbum ilustrado. Tradução. Técnicas de tradução. Tradutores em formação. Contexto universitário.

\begin{abstract}
Translating a picturebook can be a complex task because of the characteristics of this type of literary work. Issues such as the relationship between text and illustration, double page, orality, target audience, among others, are some of the challenges involved in the translation of an album. For this reason, the translator in charge of this type of translation must have the knowledge and skills required to produce a version that is adapted to the target language and culture. One way to prepare and train this professional is through a translation project that reproduces a real literary translation situation, and more particularly through the translation of picturebooks. In this article, we will study the problems encountered by a group of translation students, as well as the different approaches they adopt when translating an illustrated album from French into Spanish of a picturebook entitled Yakouba by the author-illustrator Thierry Dedieu. First, we will present the theoretical framework in relation to the main topic of our study. Subsequently, and given that this is a translation exercise in an university context, we will specify the conditions under which this activity took place and the objectives pursued with the realization of this project. Then, we will describe the main characteristics of the work to be translated and finally, we will observe the different levels at which translation difficulties may arise and the solutions proposed by the students in each case. All this will allow us to identify the most common behaviour of these translators in training and the techniques they usually apply when translating a picturebook.
\end{abstract}

Keywords: Picturebook. Translation. Translation techniques. Translators in training. University context.

RECEBIDO EM: 19 de fevereiro ACEITO EM: 04 de maio de 2019 PUBLICADO EM: julho 2019

\title{
Introduction
}

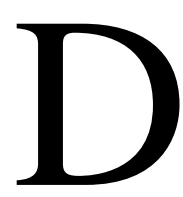

epuis la fin du XIX ${ }^{\mathrm{e}}$ siècle, dans le domaine de la littérature d'enfance et de jeunesse, la présence d'une création littéraire aux caractéristiques singulières est de plus en plus visible. Il s'agit de l'album illustré, ce nouveau modèle d'œuvre littéraire que certains considèrent comme un genre (NIÈRES-CHEVREL, 2012, p. 19-20), que d'autres conçoivent comme un « médium particulier » (VAN DER LINDEN, 2003, p. 60) et qui pour d'autres ne constitue pas un genre proprement dit mais intègre plusieurs genres (LEWIS, 2001, p. 65). Cependant, quand nous parlons d'un album illustré, nous pensons inévitablement à une image ou à une illustration. Ainsi, dans ce type de « création hybride » (CONNAN-PINTADO; GAIOTTI; POULOU, 2008, p. 8), l'illustration joue un rôle essentiel en interagissant avec le texte (VAN DER LINDEN, 2003, p. 60) pour transmettre le sens de l'histoire.

Actuellement, de nombreux axes de recherche sur l'album illustré sont explorés. Nous commencerons par examiner les aspects les plus marquants de l'album illustré afin de pouvoir nous concentrer plus tard sur le thème principal de notre étude : la traduction d'un album illustré 
dans le cadre d'un projet de formation au niveau universitaire. L'album choisi pour ce projet de traduction du français vers l'espagnol est Yakouba dont l'auteur et illustrateur est Thierry Dedieu. Ainsi, dans notre travail, nous analyserons les différentes propositions de traduction présentées par les étudiants de quatrième année du Grado en Lenguas Modernas y Traducción de l’Universidad de Alcalá en Espagne, pendant l'année universitaire 2016-2017.

A ce stade, nous pouvons émettre l'hypothèse que la traduction d'un album illustré représente des complexités supplémentaires par rapport à d'autres œuvres littéraires, et pour cette raison, la maîtrise de connaissances spécifiques dans ce domaine est indispensable pour l'accomplissement de cette tâche. Compte tenu des caractéristiques de l'album illustré, nous pensons que sa traduction peut être un défi pour le traducteur en formation qui, dans une telle situation, choisit généralement de faire une traduction littérale dans la langue cible.

Dans cette optique, les objectifs de notre étude sont les suivants : premièrement, nous essaierons de détecter les difficultés de traduction d'un album illustré et deuxièmement, nous nous proposons d'identifier les approches adoptées dans cette situation par les étudiants de traduction, considérés aussi comme des traducteurs en formation. De cette façon, nous pourrons démontrer que la traduction de cet album est une tâche complexe et que, par conséquent, une connaissance spécifique du traducteur est indispensable afin d'obtenir une version adéquate dans la langue et la culture cible.

Les pages qui suivent sont enfin le résultat d'un projet de traduction qui nous a permis de déterminer certaines des problématiques posées lors de la traduction d'un album illustré réalisée par des traducteurs en formation.

\section{Cadre théorique}

\subsection{La traduction littéraire}

La traduction littéraire consiste à traduire des textes littéraires (à savoir un roman, un conte, une poésie, etc.) d'une langue source vers une langue cible. Ce processus est délicat et comporte certaines difficultés qui doivent être prises en compte par le traducteur. Ainsi, l'objectif poursuivi dans la traduction littéraire est de produire chez le lecteur de la langue cible le même effet que chez le lecteur de la langue source, en respectant et reproduisant, dans la mesure du possible, l'intention de l'auteur de l'ouvrage d'origine. Le traducteur littéraire devra donc prendre en considération les caractéristiques inhérentes à la littéralité de ces textes : la fonction esthétique, l'approche poétique, le langage créatif et souvent subjectif, le caractère émotionnel, l'absence d'une finalité bien définie, ou en d'autres termes, la difficulté de 
déterminer, dans ce type de texte, le skopos (terme introduit par Vermeer dans les années 1970). D'après Reiss et Vermeer, la théorie du skopos fait référence à l'objectif et au but d'une traduction : "the highest rule of a theory of a translational action is the "skopos rule" : any action is determined by its purpose, i.e. it is a function of its purpose or skopos » (REISS; VERMEER, 1984/2013, p. 90).

Face à une telle situation, la traduction littéraire peut obéir à deux approches différentes. D'une part, il y a les partisans de l'application d'une traduction littérale afin de préserver le style de l'auteur et de garantir la fidélité par rapport au texte source. D'autre part, il y a ceux qui prônent une traduction plus libre dans laquelle le traducteur a le choix d'appliquer les techniques nécessaires pour obtenir une traduction convenable dans la langue cible, toujours en respectant le sens original de l'œuvre et l'intention de l'auteur.

Ces deux approches ont été analysées en traductologie. Parmi les auteurs les plus remarquables, on peut citer Nida qui a introduit les concepts d'équivalence formelle et d'équivalence dynamique utilisés dans la traduction de la Bible et qui peuvent également être appliqués dans d'autres types de traduction, comme par exemple la traduction littéraire. Pour 148 l'auteur, l'équivalence formelle consiste en une stratégie de traduction littérale qui vise à préserver les caractéristiques formelles et stylistiques du texte source : «Formal equivalence focuses attention on the message itself, in both form and content. . . One is concerned that the message in the receptor language should match as closely as possible the different elements in the source language »(NIDA, 1964, p. 159). Quant à l'équivalence dynamique, Nida et Taber proposent la définition suivante :

\footnotetext{
Dynamic equivalence is therefore to be defined in terms of the degree to which the receptors of the message in the receptor language respond to it in substantially the same manner as the receptors in the source language. This response can never be identical, for the cultural and historical settings are too different, but there should be a high degree of equivalence of response, or the translation will have failed to accomplish its purpose. (NIDA; TABER, 1969, p. 22).
}

Le principe de cette approche de traduction est d'obtenir le même effet sur le destinataire du texte dans la langue cible que sur celui du texte dans la langue source, en apportant les modifications et adaptations nécessaires à cette fin. Cependant, Nida indique qu'entre les deux extrêmes possibles (à savoir l'équivalence formelle stricte et l'équivalence dynamique complète), il existe un certain nombre de niveaux intermédiaires qui sont également acceptables en matière de traduction littéraire (NIDA, 1964, p. 160). En outre, Nida ajoute qu'une traduction réussie doit répondre à ces quatre exigences : avoir du sens, transmettre l'esprit et la forme de 
l'original, présenter une expression naturelle et simple et, surtout, produire une réponse similaire chez le destinataire cible (NIDA, 1964, p. 164).

L'approche de Nida a influencé le travail des traductologues ultérieurs. C'est le cas de l'Allemand Werner Koller qui a utilisé les théories de Nida pour étudier les différences entre les concepts d'Äquivalenz en allemand (équivalence, en français) et de Korrespondenz en allemand (correspondance, en français) dans le processus de traduction. Pour Koller le terme équivalence désignant la relation d'équivalence établie entre les textes et les contextes de la langue source et la langue cible, le terme correspondance désignant pour sa part les différences et les similitudes entre deux systèmes linguistiques (KOLLER, 1979, p. 183-185).

Enfin, Venuti mentionne à son tour deux stratégies possibles dans la traduction d'une œuvre littéraire. La première, qu'il nomme domestication en anglais (domestication, en français), consiste à adapter le texte source à la langue et à la culture cible, et la seconde, qu'il nomme foreignisation en anglais (étrangéisation, en français), implique une traduction littérale dans laquelle le style et le sens du texte source sont préservés sans adaptation à la langue et la culture cible (VENUTI, 1995, p. 20-21). Pour Venuti la stratégie d'étrangéisation est plus éthique que celle de domestication (VENUTI, 1995, p. 34). Dans le domaine de la littérature de jeunesse, Oittinen affirme que les traducteurs de ce type d'œuvres s'adressent à un public cible et adaptent le texte d'origine selon la future fonction imaginaire du texte traduit (OITTINEN, 2003, p. 129). En outre, elle ajoute que cette adaptation dans un but précis concorde avec le concept de domestication de Venuti (OITTINEN, 2003, p. 129).

\subsection{Procédés de traduction}

Les traductologues ont également identifié différentes stratégies, techniques ou procédés de traduction pouvant s'appliquer aux textes littéraires ou non littéraires. Cependant, nous adopterons ici la classification établie par Vinay et Darbelnet dans leur étude de la Stylistique comparée du français et de l'anglais en 1958 puisqu'elle constitue un point de référence dans le domaine de la traduction. Ces deux auteurs proposent ainsi sept techniques principales orientées dans deux directions possibles, à savoir la traduction directe ou littérale (procédés 1,2 et 3) et la traduction oblique (procédés 4,5,6 et 7). Rappelons ici quels sont ces sept procédés de traduction que selon Vinay et Darbelnet «toutes les grandes traductions littéraires ont reconnu implicitement »(VINAY ; DARBELNET, 1977, p. 53). 
1 Emprunt lexical : le terme de la langue source est conservé dans la langue cible.

2 Calque : type d'emprunt dont l'expression ou la structure de la langue source est traduite de manière littérale dans la langue cible.

3 Traduction littérale : traduction mot-à-mot d'un texte de la langue source vers un autre texte « correct » et « idiomatique » dans la langue cible; ce procédé est considéré comme le plus approprié surtout pour traduire entre langues de la même famille et culture.

4 Transposition : changement d'une partie du discours de la langue source par une autre dans la langue cible en conservant le même sens du message; la transposition peut être obligatoire ou facultative, selon le cas.

5 Modulation : modification dans le message qui s'obtient en changeant de point de vue ou de perspective ; le procédé de modulation peut être obligatoire ou facultative.

6 Équivalence : technique avec laquelle il est possible de rapporter la même situation dans deux langues différentes mais en utilisant des moyens stylistiques et structuraux différents ; ce procédé spécifique de traduction ne doit pas être confondu avec l'idée d'équivalence de Nida et Koller mentionnée dans les paragraphes précédents.

7 Adaptation : changement ou modification de la réalité culturelle quand la situation décrite dans la langue source n'existe pas dans la langue cible ; ce procédé est considéré comme « la limite extrême de la traduction » (VINAY; DARBELNET, 1977, p. 46-53).

Il convient aussi de noter qu'on peut employer plusieurs procédés dans une même phrase

et que, dans certains cas, il est difficile d'identifier les techniques employées dans une traduction (VINAY ; DARBELNET, 1977, p. 54).

A ces quatre procédés obliques de Vinay et Darbelnet, on pourrait en ajouter quatre autres proposés par Vazquez-Ayora en 1977 : amplification, explicitation, omission et compensation.

8 Amplification : technique qui consiste à utiliser dans la langue cible plus de monèmes que ceux présents dans la langue source pour transmettre la même idée.

9 Explicitation : type d'expansion sémantique par lequel on exprime dans la langue cible ce qui est implicite dans le contexte de la langue source.

10 Omission : suppression de certains segments jugés superflus dans la langue cible et qui étaient présents dans la langue source.

11 Compensation : méthode appliquée lors d'une perte de signification dans un segment ou dans une unité de traduction et qui doit donc être compensée dans une autre section du texte cible (VAZQUEZ-AYORA, 1977, p. 349-376).

\subsection{L'album illustré : définition, caractéristiques et traduction}

L'origine de l'album illustré remonte au début du $\mathrm{XIX}^{\mathrm{e}}$ siècle et à partir des années 1950, toute une génération d'auteurs et illustrateurs commencent à opter pour ce genre de littérature de jeunesse. D'abord, nous observons des positions différentes en ce qui concerne l'album en tant que genre littéraire. D'après Nières-Chevrel, l'album représente un genre à part entière «au même titre par exemple que le théâtre est un genre, un genre formel et susceptible [...] de décliner des sous-genres thématiques et formels » (NIÈRES-CHEVREL, 2012, p. 19- 
20). Au contraire, Lewis indique que l'album ne peut pas être considéré comme un genre à part ; en fait, il s'agit d'une production littéraire comprenant plusieurs genres (LEWIS, 2001, p. 65). Pour sa part, Van der Linden affirme que l'album constitue « une forme d'expression spécifique » et « un médium particulier » (VAN DER LINDEN, 2003, p. 59-60).

De manière générale, selon les auteurs, on observe des variations orthographiques, voire des variations de sens, en anglais. Ainsi, le terme picture book écrit en deux mots est employé par l'auteure Doonan en 1992 pour désigner un livre illustré (cité dans TABERNERO SALA, CONSEJO PANO ; CALVO VALIOS, 2015, p. 4). D'un autre côté, on trouve aussi le terme picturebook écrit comme un mot composé et proposé par exemple par Bader en 1976 ou Nikolajeva et Scott en 2001 parmi d'autres pour faire référence à un concept différent, à savoir l'album illustré.

En espagnol, le terme album ilustrado est le plus utilisé par des auteurs comme Durán en 2009 ; malgré cela, nous trouvons d'autres dénominations comme libro-album suggérée par les auteurs Colomer, Kümmerling-Meibauer et Silva-Díaz en 2010 mais aussi le terme libro ilustrado dont le sens renferme différentes nuances compte tenu de la nature des relations entre le texte, l'image et le support (TABERNERO SALA, CONSEJO PANO; CALVO VALIOS, 2015, p. 7).

En ce qui concerne la terminologie française, le mot album est le plus fréquent dans les études des auteurs comme par exemple Van der Linden en 2003 ou Nières-Chevrel en 2009. En outre, Van der Linden insiste sur le fait que l'album (illustré) ne doit pas être confondu avec le livre illustré car « il opère un renversement par rapport au livre illustré : l'image prépondérante remplace une illustration rare et subordonnée au texte » (VAN DER LINDEN, 2009, p. 2).

Comme nous pouvons le voir, il y a plusieurs façons d'aborder le concept d'album illustré ; néanmoins, nous suivrons l'approche que l'auteure Barbara Bader a introduite en 1976 :

A picturebook is text, illustrations, total design; an item of manufacture and a commercial product; a social, cultural, historical document; and, foremost, an experience for a child. As an art form it hinges on the interdependence of pictures and words, on the simultaneous display of two facing pages, and on the drama of the turning page. On its own terms its possibilities are limitless. (BADER, 1976, p. 1).

Malgré les opinions contraires, l'album présente bien des caractéristiques qui le différencient des autres genres et, notamment, il introduit « une interaction entre texte, image 
et support »(VAN DER LINDEN, 2003, p. 51). La lecture et la compréhension d'un album devront donc prendre en compte la relation établie entre l'image et le texte, tout en sachant qu'il ne s'agit pas «d'une simple co-présence mais d'une interaction du texte et des images, le sens émergeant de leurs rapports réciproques » (VAN DER LINDEN, 2003, p. 60).

En ce qui concerne la mise en page, Van der Linden considère qu'une de ses principales caractéristiques c'est que « l'organisation interne et le format de l'album montrent une grande flexibilité [...] puisqu'il n'existe pas de mise en page dite "régulière", comme dans la bande dessinée par exemple » (VAN DER LINDEN, 2009, p. 6). Malgré cette flexibilité, l'emploi de la double page est une particularité de l'album qui constitue un espace d'expression privilégié dans lequel « les images sont relativement grandes » face à « un texte relativement court » (VAN DER LINDEN, 2009, p. 6). De plus, Van der Linden ajoute l'oralité du texte comme caractéristique de l'album car il s'agit d'une œuvre qui est pensée pour être lue à haute voix (voir dans ANDERSSON ; LINDGREN, 2008, p. 26).

Une autre caractéristique des albums est qu'ils s'adressent à un public varié. Ainsi, même si un album est avant tout destiné à un public enfantin, il faut aussi considérer la 152 participation et l'intérêt du lecteur adulte dans l'acte de lecture ; nous sommes en effet face à un «double lectorat, composé de lecteurs enfants et de lecteurs adultes » (LEE, 2015, p. 54). La présence de ce double lectorat doit également être pris en compte par les éditeurs et par les auteurs lors de la traduction d'un album. Cela signifie que « les adultes, à la fois médiateurs et public cible, influencent donc tout le travail de conception de l'ouvrage traduit » (ANDERSSON et LINDGREN, 2008, p. 26). Dans leur étude sur la traduction et la réception de Pettson, de l'auteur Sven Nordqvist, en France, Andersson et Lindgren parviennent à la conclusion que les illustrations sont l'élément central au moment d'entreprendre la traduction ou la publication d'un album et elles remarquent que c'est probablement pour cette raison que les traductions des albums sont fréquemment «très libres » car la priorité est donnée aux illustrations plutôt qu'au texte (ANDERSSON ; LINDGREN, 2008, p. 27).

\section{Méthodologie}

\subsection{Le projet de traduction dans le contexte universitaire}

Dans cette étude, nous analysons la traduction d'un album illustré réalisée par des étudiants, dans un contexte universitaire. Il s'agit en réalité d'un projet de traduction pour le cours optionnel de Traducción especializada francés-español: campos y herramientas 
(Traduction spécialisée français-espagnol: domaines et outils) programmé en quatrième et dernière année de licence de Langues Modernes et Traduction.

Ce cours s'adresse habituellement à des étudiants de 21 ou 22 ans n'ayant aucune expérience professionnelle dans le secteur de la traduction ; toutefois, il peut y avoir parfois des exceptions quant à l'âge et à l'expérience préalable des étudiants puisque certains ont plus de 30 ans et ils ont déjà travaillé comme traducteurs. Dans la pratique, nous constatons que les étudiantes sont largement majoritaires dans le groupe.

Il s'agit d'une activité obligatoire dans le cadre du programme de formation de ces étudiants et, par conséquent, la traduction est corrigée et ensuite évaluée par les pairs, puis par l'enseignant. Cette tâche s'effectue par binômes, en dehors des heures de cours. Les étudiants ont un délai confortable pour la réaliser (ici, un mois). Ils peuvent utiliser les ressources qu'ils jugent appropriées et gérer le temps selon leur convenance afin d'accomplir la tâche assignée dans le délai prévu.

L'ouvrage sélectionné pour ce projet de traduction est l'album Yakouba de l'auteurillustrateur Thierry Dedieu. Le texte source est écrit en français et les étudiants doivent produire un texte cible traduit vers l'espagnol. Il est important de noter que cet album ne dispose pas encore d'une version publiée en espagnol, de sorte que les étudiants ne puissent se référer à une traduction préalable de cette œuvre. En plus de la traduction, ce projet comprenait d'autres activités complémentaires. En premier lieu, les étudiants devaient par exemple chercher des informations sur l'auteur de l'œuvre, en accordant une attention particulière à ses éventuelles publications en langue espagnole. Par la suite, ils devaient identifier et commenter les difficultés présentées par le texte de Yakouba. Finalement, chaque groupe avait à proposer une version traduite complète tout en justifiant les décisions prises pendant le processus de traduction.

Cet exercice poursuit plusieurs objectifs comme nous allons le voir à présent. Tout d'abord, l'objectif principal est de faire découvrir aux étudiants un domaine de traduction spécifique, tel que la traduction des albums illustrés, afin qu'ils puissent observer les caractéristiques de ce type de travail, les difficultés qui peuvent se présenter et les solutions envisageables.

Par ailleurs, cet exercice cherche à mettre l'étudiant dans une situation aussi proche que possible de celle qu'il pourrait trouver en travaillant en tant que traducteur professionnel. En d'autres termes, l'étudiant devra suivre les mêmes phases que dans un projet de traduction réel d'un album illustré, afin d'être conscient que les tâches d'un traducteur ne se limitent pas à la traduction. Il devra ainsi, lui aussi, accomplir d'autres opérations en aval et en amont (analyse, 
documentation, relecture, révision) qui détermineront finalement le résultat de ce processus complexe.

Cette activité a également pour but de développer la capacité d'organisation, de travail en équipe, de recherche et de réflexion sur un sujet spécifique, la capacité critique, voire d'autocritique, qui donnent à l'étudiant la compétence en traduction nécessaire dans cette profession (KELLY, 2002, p. 9). Ainsi, une fois ce travail terminé, les étudiants devront avoir acquis l'autonomie et l'expérience suffisantes pour pouvoir se débrouiller dans une situation réelle en vue de leur avenir professionnel en tant que traducteur. Ainsi et compte tenu des caractéristiques de cette tâche, nous pouvons affirmer qu'il s'agit d'une traduction professionnalisante et non d'une traduction universitaire, selon la différence que l'auteur Daniel Gile fait entre ces deux types d'orientations (GILE, 2005, p. 7-12). Enfin, rappelons que l'œuvre choisie pour ce projet n'est pas traduite en espagnol. Si la traduction obtenue est convaincante, on peut par conséquent envisager de prolonger le projet de traduction avec un projet éditorial ce qui permettrait aux étudiants d'aborder une autre facette de leur futur métier.

\subsection{Analyse des traductions de l'album}

Il est temps, à présent, de nous pencher sur l'analyse des différentes propositions de traduction de l'album Yakouba. Pour cela, nous avons suivi les étapes suivantes :

Tout d'abord, à partir des traductions que les étudiants ont faites pour leur projet, nous identifions les principaux types de difficultés qui surviennent au cours du processus de traduction. Lors de cette phase, nous nous basons sur les commentaires des étudiants concernant leurs propres difficultés, sur les corrections apportées par l'enseignant et aussi sur les difficultés que nous détectons en comparant les différentes propositions de traduction. Puis, nous nous concentrons sur les techniques et les stratégies appliquées par les étudiants pour résoudre ces complexités ; cela nous permet de détecter les techniques les plus fréquentes et les moins fréquentes, et donc le comportement caractéristique de ces traducteurs en formation face à un projet de cette nature. Par cette méthode d'analyse qualitative, nous cherchons à vérifier les hypothèses posées et à atteindre les objectifs proposés dans notre étude sur la traduction d'un album illustré dans un contexte formatif.

\subsection{Yakouba : caractéristiques de l'album}

Comme nous l'avons mentionné précédemment, le titre de l'album est Yakouba de l'auteur et illustrateur français, Thierry Dedieu. Il s'agit d'un album illustré relativement court, 
soit 40 pages, sans pagination interne, de forme rectangulaire qui est disponible avec une couverture rigide ou format poche souple. L'ouvrage présente une combinaison de texte et d'images qui forment une œuvre « hybride » (VAN DER LINDEN, 2003, p. 52) caractérisée par une prédominance des illustrations sur le texte. La distribution entre le texte et les images n'est pas homogène, car parfois l'image apparaît d'abord sur la page gauche, puis le texte sur la page droite, et parfois c'est l'inverse. Nous apprécions également qu'à trois reprises, une image occupe une double page, et qu'à une seule occasion, le texte occupe deux pages sans inclure aucune image. Néanmoins, il se confirme que les illustrations jouent un rôle protagoniste dans le développement de l'histoire, tandis que le texte, de plus petite taille et avec une extension limitée, est perçu comme un élément secondaire mais en même temps nécessaire pour la configuration de l'intrigue.

L'emplacement du texte par rapport à l'image ne suit pas de critères puisque, parfois, un fragment de texte en blanc apparait isolé sur une page noire, alors que, d'autres fois, un texte en noir est inséré dans l'image ; ceci correspond aux termes proposés par Van der Linden, à savoir extraiconique (texte hors image) et intraiconique (texte intégré dans l'illustration) (cité dans ANDERSSON ; LINDGREN, 2008, p. 27). Nous pensons également qu'il y a une relation de complémentarité entre le texte et l'image qui donnent du sens à l'histoire (VAN DER LINDEN, 2008, p. 53) et pour cette raison, une interprétation globale est nécessaire de la part du lecteur.

En ce qui concerne les illustrations, plusieurs aspects méritent une attention particulière. D’une part, les deux seules couleurs présentes sont le blanc et le noir, dans des intensités différentes. Le style figuratif utilisé dans les illustrations facilite l'identification et l'interprétation subséquente des scènes représentées. Pour donner forme aux illustrations, le dessinateur utilise la technique du dessin au fusain et pastels gras (METTOUDI; BUFFIERE DELAIR, 2011, p. 105). De plus, on remarque la tendance de l'auteur à capturer la perspective et à jouer avec le contraste des noirs et des blancs présents dans l'album.

L'album a recours à un titre éponyme, l'auteur utilisant le nom du personnage principal, Yakouba, un enfant qui vit dans un petit village en Afrique avec sa famille et d'autres habitants. L'album commence par une description de l'environnement dans lequel se déroule l'action, c'est-à-dire le petit village africain où tous se préparent pour le grand jour : les enfants vont devenir adultes en devenant chasseurs. En réalité, il n’y a pas de description spécifique des personnages, mais l'unité composée du texte et des images aide le lecteur à se construire sa propre perception de ceux-ci. Pour faire preuve de son courage, Yakouba doit tuer un lion. Mais 
ce lion, qui acquiert sa propre voix dans l'histoire, est blessé et fatigué, et il le fait savoir à Yakouba à travers son regard. Ainsi, dès que l'enfant et le lion croisent leur regard, leur destin change complètement. Yakouba doit alors décider alors s'il va tuer le lion blessé et fatigué pour devenir un guerrier courageux ou si, au contraire, il épargnera l'animal au risque d'être rejeté par son peuple. Et voici l'histoire d'un enfant qui ne devient finalement pas un guerrier courageux. A travers cette relation établie entre l'enfant, qui symbolise l'être humain, et le lion, qui représente l'animal sauvage, le lecteur est invité à réfléchir sur des aspects tels que le courage, l'honneur et le déshonneur, la valeur des traditions, l'empathie, la justice et la morale, entre autres.

Par conséquence, il faut considérer que toutes ces caractéristiques et particularités de l'album peuvent constituer une difficulté supplémentaire en matière de traduction. Pour cette raison, et afin de fournir une traduction qui fonctionne dans la langue et la culture cible, le traducteur devra non seulement traduire, mais aussi adapter ou créer un texte à partir de mots, images ou idées en appliquant les techniques appropriées dans chaque cas (GRANDE, 2014).

\section{La traduction de l'album : difficultés et solutions}

Nous avons observé que, pendant la traduction de l'album Yakouba, les étudiants ont affronté différents types de difficultés et pour les surmonter, ils ont dû mettre en pratique leurs compétences et leurs connaissances en traduction afin de trouver la solution la plus appropriée dans chaque cas. Voici un aperçu des difficultés rencontrées et des solutions choisies. Nous avons classé les premières en fonction de leur nature : lexicale et sémantique, syntactique, grammaticale, phonétique, rhétorique et pragmatique.

\section{Difficultés au niveau lexical et sémantique}

Les mots polysémiques sont inclus dans cette catégorie. Un exemple de ce phénomène est le verbe «se parer» dans l'expression « On se maquille, on se pare ». Dans ce cas, il s'agit d'un verbe qui présente plusieurs sens et c'est pour cette raison qu'il peut poser un problème lors de sa traduction. Cette difficulté peut être résolue à l'aide du contexte et de l'illustration de la page suivante sur laquelle on peut voir une femme vêtue d'une tenue africaine traditionnelle, avec un turban sur la tête et d'autres accessoires tels que boucles d'oreilles, bracelets et colliers. Malgré les multiples traductions possibles du même verbe, nous constatons que la plupart des groupes ont choisi d'utiliser en espagnol le verbe engalanarse, qui est considéré comme un 
choix approprié dans la langue cible et selon ce contexte particulier : «La gente se maquilla, se engalana ».

L'expression «par tes pairs » constitue un autre cas de difficulté sémantique dans la phrase « tu sors grandi, mais banni tu le seras par tes pairs ». La plupart des étudiants ont ainsi décidé de la traduire en utilisant la technique de la traduction littérale sans trop s'écarter du terme original, ce qui a abouti à une traduction peu naturelle et confuse dans la langue cible comme le montre l'exemple en espagnol «por tus iguales».

Enfin, dernier exemple de difficulté lexicale et sémantique, l'expression « se sentir rocher » dans la phrase « Sous un soleil de plomb, marcher, franchir les ravins, contourner les collines, se sentir rocher, forcément, [...] » a compliqué le processus de traduction. Il s'agit d'une structure inhabituelle dans le langage courant mais qui peut être caractéristique du langage littéraire. L'auteur utilise cette expression pour comparer Yakouba avec un élément de la nature, le rocher, afin de transmettre au lecteur l'image de la situation dans laquelle se trouvait l'enfant à ce moment précis, c'est-à-dire tous les obstacles à surmonter pour trouver et affronter le lion. Une fois de plus, nous observons une tendance à la traduction littérale, dans ce cas par le procédé du calque, c'est-à-dire en utilisant la même structure et la traduction littérale de ces mots en espagnol, «sentirse roca ». Cependant, cette stratégie ne fonctionne pas toujours dans la langue cible, et notamment ici car il s'agit d'un texte littéraire dans lequel l'expression est utilisée dans un sens métaphorique. D'autre part, nous constatons que certains étudiants ont utilisé d'autres techniques telles que l'amplification «sentirse como una roca » ou la modulation en combinaison avec l'explicitation «camuflarse, necesariamente, con las rocas » afin d'essayer de refléter le sens que l'auteur voulait transmettre avec ces mots.

\section{Difficultés au niveau syntactique}

Prenons par exemple l'utilisation de la voix passive en français et sa traduction en espagnol. Dans notre album, on trouve la phrase suivante à la voix passive « le bétail ne fut plus jamais attaqué par les lions ». Or nous observons qu'elle a été abordée de deux façons possibles. En général, les étudiants ont choisi la traduction rétablissant la voix active car elle est plus naturelle et fréquente en espagnol et ils ont ainsi eu recours à des procédés telles que la modulation dans la traduction vers l'espagnol «los leones nunca más atacaron al ganado ». D'autre part, un plus petit nombre d'étudiants ont préféré conserver la voix passive en espagnol et appliquer une traduction plus littérale donnant lieu à la version suivante, « el rebaño dejó de ser atacado por los leones ». Dans ce cas, les deux possibilités sont acceptées bien que nous 
partagions l'avis de la majorité des étudiants qui ont choisi de changer la phrase à la voix active afin de l'adapter aux usages les plus courants de la langue espagnole.

Dans la phrase « à tes propres yeux, tu sors grandi, mais banni, tu le seras par tes pairs » que nous avons déjà citée, nous trouvons un autre exemple de difficulté syntactique qui consiste à modifier l'ordre dans la phrase. L'auteur utilise cette ressource dans le texte source pour créer une rime et pour embellir la langue à l'aide d'une hyperbate. La plupart des groupes ont réalisé une version plus libre et ont adapté l'ordre des mots espagnols par des techniques telles que la modulation ou la transposition pour obtenir une traduction reproduisant un ordre des mots plus fréquent dans le langage courant, comme dans la phrase de l'un des groupes : « habrás madurado a tus propios ojos, pero tus semejantes te desterrarán ». Certains étudiants ont pourtant choisi de conserver l'ordre des mots du texte source dans leur proposition de traduction : " a tus propios ojos sales engrandecido, pero rechazado serás por tus iguales ». Cependant, nous voyons qu'il est difficile de garder la rime en espagnol surtout si on opte pour une traduction littérale. Cet exemple présente non seulement une difficulté au niveau syntactique mais aussi une difficulté au niveau rhétorique car il implique l'utilisation de

158 l'hyperbate (une figure rhétorique) et de la rime.

\section{Difficultés au niveau grammatical}

Plusieurs groupes ont indiqué que l'une des difficultés à laquelle ils avaient dû faire face est la traduction des temps et des formes verbales du français vers l'espagnol. Dans le cas de l'expression «s'armer de courage et s'élancer pour combattre » nous avons ainsi détecté que les étudiants préfèrent garder la même forme verbale à l'infinitif et traduire de façon littérale vers l'espagnol, c'est-à-dire «armarse de valor y lanzarse al combate ». On observe cette approche à plusieurs reprises en présence de verbes à l'infinitif dans le texte source. Néanmoins, d'autres groupes ont préféré d'autres stratégies comme par exemple le changement du temps du verbe de l'infinitif au passé (à savoir le pretérito perfecto simple de indicativo en espagnol) : " se armó de valor y se lanzó al combate »; une autre proposition était de changer l'infinitif par l'indicatif présent (presente del indicativo en espagnol) : "se arma de valor y se lanza al combate ». Par conséquence, nous pensons que dans ce cas, il est préférable de conserver l'infinitif dans la traduction, en tenant compte du fait que les verbes qui apparaissent avant et après cette expression sont également à l'infinitif. De plus, de cette manière il est possible de respecter ainsi le fil des événements dans cette histoire. 


\section{Difficultés au niveau phonétique}

Une autre difficulté concerne la traduction du nom du personnage principal et du titre du livre, Yakouba. Nous voyons que la tendance des étudiants dans cette situation est de ne pas modifier le nom et d'appliquer la technique d'emprunt dans la version espagnole, c'est-à-dire en gardant le nom Yakouba. Cependant, si on s'intéresse à la prononciation du nom en français, on constate que la graphie du groupe de lettres « ou » représente le phonème /u/ en français. De même, nous tenons à rappeler que l'une des caractéristiques d'un album est l'oralité du texte et, par conséquent, cela implique que le nom Yakouba doit être adapté à la prononciation dans la langue cible. De cette façon, et afin d'obtenir la même prononciation en espagnol, il serait nécessaire d'adapter l'écriture du nom en changeant le groupe de lettres « ou » pour la lettre « $\mathrm{u} \gg$ en écrivant le nom Yakuba en espagnol.

\section{Difficultés au niveau rhétorique}

Les étudiants ont également éprouvé des difficultés à traduire des figures de style, comme par exemple l'asyndète dans les lignes suivantes : «Sous un soleil de plomb, marcher, franchir les ravins, contourner les collines, se sentir rocher, forcément, herbe, bien sûr, vent, certainement, eau, très peu ». La complexité de la structure syntaxique s'observe dans une énumération de verbes suivis d'articles et de noms, qui est finalement réduite à une série de noms et d'adverbes séparés par des virgules avec une absence de connecteurs et de conjonctions. Ces figures rhétoriques sont fréquentes dans le langage littéraire et sont souvent utilisées dans le but d'intensifier le caractère expressif du message. Une des solutions proposées par les étudiants était de répéter la même structure et de réaliser une traduction littérale dans la langue cible, comme on peut le voir dans cette phrase en espagnol : «Bajo un sol abrasador, caminar, cruzar los barrancos, rodear las colinas, sentirse como una roca, es necesario, como la hierba, seguro, como el viento, cierto, como el agua, insuficiente ». Une autre solution adoptée par les étudiants a été d'essayer une traduction plus libre en espagnol en utilisant plusieurs techniques en même temps telles que l'adaptation et l'amplification comme dans le cas suivant : «Bajo un sol abrasador, hay que caminar, atravesar barrancos, rodear colinas. Hay que sentirse fuerte como una roca, no queda otra. Hierba hay, por supuesto, también viento. Agua... muy poca». Par conséquent, nous pensons que face à ce type de difficulté de traduction de figures de style, il convient d'appliquer les techniques nécessaires (par exemple, l'adaptation, l'amplification, etc.) pour obtenir une version adaptée et adéquate dans la langue cible. 


\section{Difficultés au niveau pragmatique}

Des difficultés ont également été rencontrées à ce niveau, notamment dans la traduction de la phrase : «Tu n'aurais donc aucun mal à venir à bout de mes forces ». Plusieurs étudiants ont admis que la traduction de la locution verbale venir à bout de composée d'un verbe, d'une préposition, d'un nom et d'une autre préposition dont le sens dans ce contexte est vaincre, avait été un défi et que, par conséquent, ils avaient dû recourir à différentes stratégies ou techniques pour trouver une solution à ce problème.

Une fois le sens de l'expression compris, nous constatons effectivement que plusieurs possibilités ont été envisagées pour la traduction en espagnol. Une des solutions trouvées a été d'employer simultanément les procédés de modulation et d'omission : «Por lo tanto, podrías derrotarme con facilidad». Un autre groupe a estimé que la solution la plus efficace était de traduire la locution d'une manière plus libre avec le procédé de modulation : "No tendrías problema en acabar con las pocas fuerzas que me quedan ». Dans ce cas, nous pouvons affirmer que les étudiants ont préféré éviter la traduction littérale de l'expression et ont opté pour une traduction plus libre en appliquant les stratégies qu'ils ont estimées pertinentes pour obtenir une

160 version adaptée en espagnol. Cet exemple montre que les étudiants sont conscients de ces problématiques liées à la traduction d'un album et que, dans des cas comme celui-ci, ils décident de «prendre des risques » en se détachant du texte source afin de fournir une traduction efficace dans la langue et la culture cible.

Ainsi, avec ces exemples, nous avons essayé d'expliquer les difficultés les plus fréquentes lors de la traduction d'un album illustré. Cependant, il faut tenir compte du fait que chaque album peut avoir ses propres caractéristiques et donc des problèmes de traduction spécifiques. D'autre part, nous avons constaté que les étudiants ont été capables de réagir à cette situation en proposant diverses approches. Cependant, ils ont opté à plusieurs reprises pour une traduction littérale, ce qui a abouti en une traduction orientée vers la langue et la culture source plutôt que vers la langue, la culture et le destinataire cible et donc contrairement à la tendance observée en traduction d'ouvrages pour la jeunesse.

\section{En guise de conclusion}

La traduction d'un album peut sembler facile à première vue, mais nous avons pu vérifier qu'il s'agit en réalité d'une tâche complexe en raison des caractéristiques de ce type d'œuvre et qui représentent des difficultés supplémentaires pendant le processus de traduction dans une autre langue, à savoir la relation entre texte e image, le double lectorat, l'organisation 
interne, la fonction d'oralité, etc. Par conséquent, nous pouvons affirmer que le traducteur qui fait face à ce type de traduction requiert une formation spécialisée et/ou une expérience préalable dans ce domaine spécifique.

Comme nous l'avons vu dans notre analyse, les étudiants ou traducteurs en formation ont été capables d'identifier les difficultés - lexicales, sémantiques, syntactiques, grammaticales, phonétiques, rhétoriques et pragmatiques, entre autres - liées à la traduction de l'album. Et certains ont su utiliser leurs connaissances et leurs compétences en traduction pour trouver une solution adaptée dans chaque cas. Ils ont aussi eu recours à des procédés tels que la modulation, la transposition, l'omission et l'amplification.

Cependant, nous avons observé que la plupart optent pour une traduction littérale avec l'intention de respecter, autant que possible, le sens et la structure du texte source, peut-être par peur de prendre des risques.

Enfin, étant donné que nous avons analysé la traduction d'un seul album illustré, les résultats obtenus peuvent être considérés comme modestes. Cependant, nous espérons que notre travail contribuera à faire prendre conscience de la complexité de la traduction de ce type d'ouvrage et de la nécessité d'une formation spécialisée dans ce domaine afin que les futurs traducteurs puissent accomplir leur travail de manière efficace.

\section{REFERENCES BIBLIOGRAPHIQUES}

ANDERSSON, Carina; LINDGREN, Charlotte. Texte, image et désignateurs culturels : réflexions sur la traduction et la réception de Pettson en France. Moderna Språk, v. 2, p. 2434, 2008.

BADER, Barbara. American Picturebooks from Noah's Ark to the Beast Within. New York : Macmillan Publishing Company, 1976.

COLOMER, Teresa; KÜMMERLING-MEIBAUER, Bettina; SILVA-DÍAZ, María C.. Cruce de miradas : Nuevas aproximaciones al libro-álbum. Barcelona : Banco del LibroGRETEL, 2010.

CONNAN-PINTADO, Christiane ; GAIOTTI, Florence; POULOU, Bernadette. L'album contemporain pour la jeunesse : nouvelles formes, nouveaux lecteurs ? Modernités 28, Presses Universitaires de Bordeaux, 2008.

DEDIEU, Thierry. Yakouba. Paris : Seuil Jeunesse, 2015.

DOONAN, Jane. Looking at Pictures in Picture Books. Stroud : Thimble Press, 1992. 
DURÁN, Teresa. Álbumes y otras lecturas : análisis de los libros infantiles. Barcelona : Octaedro, 2009.

GILE, Daniel. La traduction. La comprendre, l'apprendre. Paris : Presses Universitaires de France, 2005.

GRANDE, Mario. Traducir literatura infantil y juvenil : Notas a vuelapluma. El Trujamán. Revista diaria de traducción, 7 jan. 2014.

KELLY, Dorothy A. Un modelo de competencia traductora : bases para el diseño curricular. Puentes. Hacia nuevas investigaciones en la mediación intercultural, n. 1, 2002, p. 9-20.

KOLLER, Werner. Einführung in die Übersetzungswissenschaft. Heidelberg : Quelle \& Meyer, 1979.

LEE, Sungyup. Intertextualité dans la traduction des albums de type "double lectorat". »Meta, Montreal, v. 60, n. 1, p. 53-70, 2015.

LEWIS, David. Reading Contemporary Picturebooks: Picturing Text. New York : Routledge/Falmer, 2001.

METTOUDI, Chantal; DELAIR, Maryse Buffière. Comment enseigner... Les activités artistiques en maternelle. Paris : Hacette Éducation, 2011.

NIDA, Eugene A. Toward a Science of Translating: With Special Refference to Principles and Procedures Involved in Bible Translating. Leiden : E. J. Brill, 1964.

NIDA, Eugene A.; TABER, Charles R.. The Theory and Practice of Translation. Leiden : E. J. Brill, 1969.

NIERES-CHEVREL, Isabelle. L'album, le mot, la chose. L'album, le parti pris des images, Ed. Viviane Alary et Nelly Chabrol-Gagne. Clermont-Ferrand : Presses universitaires Blaise Pascal, 2012, p. 15-20.

NIERES-CHEVREL, Isabelle. Introduction à la littérature de jeunesse. Paris : Didier Jeunesse, 2009.

NIKOLAJEVA, Maria; SCOTT, Carole. How Picturebooks Work. New York : Garland, 2001.

OITTINEN, Riitta. Where the Wild Things Are : Translating Picture Books. Meta, Montreal, v. 48, n. 1-2, p. 128-141, 2003.

REISS, Katharina, VERMEER, Hans J.. Towards a General Theory of Translational Action. Skopos Theory Explained. Traduit par Christiane Nord. Manchester : St Jerome, 1984/2013.

TABERNERO SALA, Rosa; CONSEJO PANO, Elena, CALVO VALIOS, Virginia . LIJ ilustrada : dificultades en la traducción de los conceptos que la definen. Introducción. 
Perspectivas multifacéticas en el universo de la literatura infantil y juvenil. Ed. Gloria Bazzocchi, Pilar Capanaga, et Raffaella Tonin. Mediazioni, n. 17, 2015.

VAN DER LINDEN, Sophie. «L'album, entre texte, image et support. » La Revue des livres pour enfants, n. 214, p. 59-68, 2003.

VAN DER LINDEN, Sophie. L'album, un support artistique ? La littérature de jeunesse en question (s). Ed. Nathalie Prince. Rennes : Presses universitaires de Rennes, 2009.

VAN DER LINDEN, Sophie. L'album, le texte et l'image. Le français aujourd'hui, v. 161, n. 2, p. 51-58, 2008.

VAZQUEZ-AYORA, Gerardo. Introducción a la Traductología : Curso Básico de Traducción. Washington : Georgetown University Press, 1977.

VENUTI, Lawrence. The Translator's Invisibility : A History of Translation. London : Routledge, 1995.

VINAY, Jean-Paul; DARBELNET , Jean. Stylistique comparée du français et de l'anglais. Paris : Didier, 1977.

\footnotetext{
* Raymonda NODIS - Master en Communication Interculturelle, Interprétation et Traduction dans les Services Publics (2018) et Licence en Langues Modernes et Traduction (2016) à l'Université d'Alcalá (Madrid, Espagne). Chercheuse à l'Université d'Alcalá grâce à une bourse de recherche dans le domaine de la traduction (2018-2019). Alcalá de Henares, Espagne.

E-mail: rayda.nodis@gmail.com

ORCID: https://orcid.org/0000-0003-1352-4455
} 